\title{
The Invariant Variational Bicomplex
}

\author{
Irina A. Kogan and Peter J. Olver
}

\begin{abstract}
We establish a group-invariant version of the variational bicomplex that is based on a general moving frame construction. The main application is an explicit group-invariant formula for the Euler-Lagrange equations of an invariant variational problem.
\end{abstract}

\section{Introduction.}

Most modern physical theories begin by postulating a symmetry group and then formulating field equations based on a group-invariant variational principle. As first recognized by Sophus Lie, [17], every invariant variational problem can be written in terms of the differential invariants of the symmetry group. The associated Euler-Lagrange equations inherit the symmetry group of the variational problem, and so can also be written in terms of the differential invariants. Surprisingly, to date no-one has found a general group-invariant formula that enables one to directly construct the Euler-Lagrange equations from the invariant form of the variational problem. Only a few specific examples, including plane curves and space curves and surfaces in Euclidean geometry, are worked out in Griffiths, [12], and in Anderson's notes, $[\mathbf{3}]$, on the variational bicomplex.

In this note, we summarize our recent solution to this problem; full details can be found in [16]. Our principal result is that, in all cases, the Euler-Lagrange equations have the invariant form

$$
\mathcal{A}^{*} \mathcal{E}(\widetilde{L})-\mathcal{B}^{*} \mathcal{H}(\widetilde{L})=0,
$$

where $\mathcal{E}(\widetilde{L})$ is an invariantized version of the usual Euler-Lagrange expression or "Eulerian", while $\mathcal{H}(\widetilde{L})$ is an invariantized Hamiltonian, which in the multivariate context is, in fact, a tensor, $[\mathbf{2 0}]$, and $\mathcal{A}^{*}, \mathcal{B}^{*}$ certain invariant differential operators, which we name the Eulerian and Hamiltonian operators. Our methods produce an explicit computational algorithm for determining the invariant differential operators $\mathcal{A}^{*}, \mathcal{B}^{*}$, that, remarkably, can be directly constructed from the formulae for the infinitesimal generators of the transformation group action using only linear algebra and differentiation.

1991 Mathematics Subject Classification. 53A55, 58J70, 58E40, 35A30, 49S05, 58A20.

Supported in part by NSF Grant DMS 99-83403.

Supported in part by NSF Grant DMS 98-03154. 
This result will be based on combining two powerful ideas in the modern, geometric approach to differential equations and the variational calculus. The first is the variational bicomplex, due to Vinogradov, [23, 24], and Tulczyjew, [22], of fundamental importance in the study of the geometry of jet bundles, differential equations and the calculus of variations. Later contributions of Tsujishita, [21], and Anderson, $[\mathbf{1}, \mathbf{3}]$, have amply demonstrated the power of the bicomplex formalism for both local and global problems in the geometric theory of differential equations and the calculus of variations.

The second ingredient is an equivariant reformulation of Cartan's moving frame theory, $[\mathbf{6}, \mathbf{1 1}, \mathbf{1 3}, \mathbf{1 5}]$, due to the second author and Fels, $[\mathbf{9}, \mathbf{1 0}]$. For a general finite-dimensional transformation group $G$, a moving frame is defined as an equivariant map from an open subset of jet space to the Lie group $G$. Once a moving frame is established, it provides a canonical mechanism, called invariantization, that allows us to systematically construct the invariant counterparts of all objects of interest in the usual variational bicomplex, including differential invariants, invariant differential forms, etc. The key formula relates the differentials of ordinary functions and forms to the invariant differentials of invariant functions and forms, which requires additional "correction terms" similar to the terms that distinguish covariant derivatives in Riemannian geometry from ordinary derivatives. In particular, these formulae form the basis for a complete classification of the syzygies and commutation formulae for differentiated invariants, as first discovered in [10]. The invariant version of the vertical bicomplex differential will then produce the desired formula relating invariant variational problems and Euler-Lagrange equations.

The final formula is not elementary; nevertheless, an explicit computational algorithm based only on infinitesimal data, linear algebra and differentiation is established, which allows one to treat very general transformation groups with the aid of a standard computer algebra system. Our own computations have been implemented in both MATHEMATiCA and MAPLE, and the results compared in order to give added assurance of their overall correctness. We also note that the MAPLE software package VESSIOT, [2], developed by Ian Anderson and his students, provides an extensive collection of general purpose routines for performing complicated bicomplex constructions. A number of the moving frame algorithms have been successfully implemented using the VESSIOT package. We also note that V. Itskov has recently proposed an alternative foundation of the subject, based on a new approach to symmetry reduction of exterior differential systems and variational problems; see $[\mathbf{1 4}]$ and his contribution to these proceedings.

\section{The Invariant Variational Bicomplex.}

We begin with a brief review of the variational bicomplex, relying primarily on the lucid presentation in $[\mathbf{3}, \mathbf{2 1}]$. See also [18] for basic results on jet bundles, contact forms, prolongation, etc. Given an $m$-dimensional manifold $M$, we let $\mathrm{J}^{n}=\mathrm{J}^{n}(M, p)$ denote the $n^{\text {th }}$ order (extended) jet bundle consisting of equivalence classes of $p$-dimensional submanifolds $S \subset M$ under the equivalence relation of $n^{\text {th }}$ order contact. The infinite jet bundle $\mathrm{J}^{\infty}=\mathrm{J}^{\infty}(M, p)$ is defined as the inverse limit of the finite order jet bundles under the standard projections $\pi_{n}^{n+1}: \mathrm{J}^{n+1} \rightarrow \mathrm{J}^{n}$. Differential functions, meaning functions $F: \mathrm{J}^{n} \rightarrow \mathbb{R}$ defined on an open subset of jet space, and differential forms on $\mathrm{J}^{n}$ will be routinely identified with their 
pull-backs to the appropriate open subset of the infinite jet space. When we introduce local coordinates $z=(x, u)$ on $M$, we consider the first $p$ components $x=\left(x^{1}, \ldots, x^{p}\right)$ as independent variables, and the latter $q=m-p$ components $u=\left(u^{1}, \ldots, u^{q}\right)$ as dependent variables. The induced coordinates on the jet bundle $\mathrm{J}^{\infty}$ are denoted by $z^{(\infty)}=\left(x, u^{(\infty)}\right)$, consisting of independent variables $x^{i}$, dependent variables $u^{\alpha}$, and their derivatives $u_{J}^{\alpha}, \alpha=1, \ldots, q, 0<\# J$, of arbitrary order. Here $J=\left(j_{1}, \ldots, j_{k}\right)$, with $1 \leq j_{\nu} \leq p$, is a symmetric multi-index of order $k=\# J$.

A differential form $\theta$ on $\mathrm{J}^{\infty}$ is called a contact form if it is annihilated by all jets, so that $\theta \mid \mathrm{j}_{\infty} S=0$ for every $p$-dimensional submanifold $S \subset M$. The contact or vertical subbundle $\mathcal{C}^{(\infty)} \subset T^{*} \mathrm{~J}^{\infty}$ is spanned by the contact one-forms. In local coordinates, every contact one-form is a linear combination of the basic contact forms

$$
\theta_{J}^{\alpha}=d u_{J}^{\alpha}-\sum_{i=1}^{p} u_{J, i}^{\alpha} d x^{i}, \quad \alpha=1, \ldots, q, \quad 0 \leq \# J .
$$

On the other hand, the coordinate one-forms $d x^{1}, \ldots, d x^{p}$ span the complementary horizontal subbundle $\mathrm{H} \subset T^{*} \mathrm{~J}^{\infty}$. The splitting $T^{*} \mathrm{~J}^{\infty}=\mathrm{H} \oplus \mathcal{C}^{(\infty)}$ of the cotangent bundle induces a bi-grading $\Omega^{*}=\bigoplus \Omega^{r, s}$ of the space of all differential forms on $\mathrm{J}^{\infty}$, with $\pi_{r, s}: \Omega^{*} \rightarrow \Omega^{r, s}$ the projection to the space of forms of bigrade $(r, s)$, which are linear combinations of wedge products of $r$ horizontal forms $d x^{i}$ and $s$ contact forms $\theta_{K}^{\alpha}$. The differential on $\mathrm{J}^{\infty}$ splits into horizontal and vertical components, $d=d_{H}+d_{V}$, where $d_{H}: \Omega^{r, s} \rightarrow \Omega^{r+1, s}$ increases horizontal degree, and $d_{V}: \Omega^{r, s} \rightarrow \Omega^{r, s+1}$ increases vertical degree. Closure, $d \circ d=0$, implies that $d_{H} \circ d_{H}=0=d_{V} \circ d_{V}$, while $d_{H} \circ d_{V}=-d_{V} \circ d_{H}$. The resulting structure is known as the variational bicomplex.

Given an $r$-dimensional Lie group $G$ act smoothly on the manifold $M$, we let $G^{(n)}$ denote the $n^{\text {th }}$ prolongation of $G$ to the jet bundle $\mathrm{J}^{n}=\mathrm{J}^{n}(M, p)$ induced by the action of $G$ on $p$-dimensional submanifolds. If $G$ acts effectively on each open subset of $M$ then, due to the stabilization theorem [19], the prolonged action becomes locally free on a dense open subset $\mathcal{V} \subset \mathrm{J}^{\infty}$, known as the regular subset, and hence, $[\mathbf{8}]$, one can find a (locally) invariant coframe, and thus an invariant version of the variational bicomplex.

As in [10], we introduce the lifted jet bundle $\mathcal{B}^{\infty}=G \times \mathrm{J}^{\infty}$, along with the regularized prolonged group action $g \cdot\left(h, z^{(\infty)}\right)=\left(h \cdot g^{-1}, g^{(\infty)} \cdot z^{(\infty)}\right)$. The components of the evaluation map $w\left(g, z^{(\infty)}\right)=g^{(\infty)} \cdot z^{(\infty)}$ provide a complete system of lifted differential invariants on $\mathcal{B}^{\infty}$. This endows the bundle

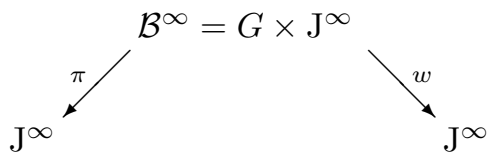

with a double fibration structure. The Cartesian product structure on $\mathcal{B}^{\infty}$ induces a bigrading $\widehat{\Omega}^{*}=\bigoplus \widehat{\Omega}^{r, s}$ of the space of differential forms on $\mathcal{B}^{\infty}$, where $\widehat{\Omega}^{r, s}$ is the space of differential forms which are linear combinations of wedge products of $r$ jet forms $d x^{i}, \theta_{K}^{\alpha}$ and $s$ Maurer-Cartan forms $\mu^{\kappa}$. We accordingly decompose the differential $d=d_{J}+d_{G}$ into jet and group components, which forms a trivial product bicomplex structure on $\mathcal{B}^{\infty}$. Let $\widehat{\Omega}_{J}^{*}=\oplus \widehat{\Omega}^{r, 0}$ denote the space of pure jet forms, i.e., linear combinations of wedge products of $d x^{i}, \theta_{K}^{\alpha}$ only, whose coefficients 
may depend on jet coordinates and group parameters. The jet projection $\pi_{J}: \widehat{\Omega}^{*} \rightarrow$ $\widehat{\Omega}_{J}^{*}$ annihilates all the Maurer-Cartan forms.

Local freeness of the prolonged action implies that one can construct a right (left) moving frame on the regular subset $\mathcal{V} \subset \mathrm{J}^{\infty}$, that is, a locally right (left) $G$-equivariant map $\rho: \mathcal{V} \rightarrow G$. A moving frame defines a $G$-equivariant section $\sigma: \mathcal{V} \rightarrow \mathcal{B}^{\infty}$, namely $\sigma\left(z^{(\infty)}\right)=\left(\rho\left(z^{(\infty)}\right), z^{(\infty)}\right)$. See $[\mathbf{1 0}, \mathbf{1 6}]$ for the computational algorithm for explicitly constructing a moving frame, based on Cartan's method of normalization, [6], which amounts to a choice of cross-section to the group orbits. The most important definition in this paper tells us how to "invariantize" an arbitrary differential form using the moving frame section.

Definition 1. The invariantization of a differential form $\Omega$ on $\mathrm{J}^{\infty}$ is the invariant differential form $\iota(\Omega)=\sigma^{*}\left(\pi_{J}\left(w^{*} \Omega\right)\right)$.

Invariantization defines a canonical projection (depending upon the moving frame) from the space of differential forms to the space of invariant differential forms. In particular the invariantized coordinate functions provide a complete system of differential invariants,

$$
\begin{aligned}
H^{i}\left(x, u^{(n)}\right) & =\iota\left(x^{i}\right), & & i=1, \ldots, p, \\
I_{K}^{\alpha}\left(x, u^{(l)}\right) & =\iota\left(u_{K}^{\alpha}\right), & & \alpha=1, \ldots, q, \quad k=\# K \geq 0,
\end{aligned}
$$

known as the normalized differential invariants. Invariantization of the basis oneforms $d x^{i}, \theta_{K}^{\alpha}$ provides an invariant coframe

$$
\begin{aligned}
\varpi^{i} & =\iota\left(d x^{i}\right), & & i=1, \ldots, p, \\
\vartheta_{K}^{\alpha} & =\iota\left(\theta_{J}^{\alpha}\right), & & \alpha=1, \ldots, q,
\end{aligned}
$$

The $\vartheta_{K}^{\alpha}$ form an invariant basis for the space of contact one-forms, while the invariant horizontal forms decompose, $\varpi^{i}=\omega^{i}+\eta^{i}$, as a sum of the usual contactinvariant horizontal forms $\omega^{i} \in \Omega^{1,0},[\mathbf{1 0}, \mathbf{1 8}]$, (not to be confused with invariant contact forms), along with additional contact "corrections" $\eta^{i} \in \Omega^{0,1}$ so as to make the $\varpi^{i}$ fully invariant one-forms. If $G$ acts projectably, there are no contact corrections: $\eta^{i}=0$. The total vector fields $\mathcal{D}_{1}, \ldots, \mathcal{D}_{p}$ dual to $\omega^{1}, \ldots, \omega^{p}$ form a complete set of invariant differential operators that map differential invariants to differential invariants, and, more generally, invariant differential forms to invariant differential forms by Lie differentiation, denoted $\mathcal{D}_{i}(\Omega)$. The invariant coframe $\varpi^{i}, \vartheta_{J}^{\alpha}$ is used to bigrade the space of differential forms $\Omega=\oplus \widetilde{\Omega}^{r, s}$ on $\mathrm{J}^{\infty}$. If the group acts non-projectably, the invariant bigradation is different from the standard bicomplex bigradation, $\Omega^{r, s} \neq \widetilde{\Omega}^{r, s}$.

The most important fact underlying the general construction is that the invariantization map $\iota$ does not respect the exterior derivative operator. Thus, in general, $d \iota(\Omega) \neq \iota(d \Omega)$. The recurrence formulae, $[\mathbf{1 0}, \mathbf{1 6}]$, provide the missing "correction terms" $d \iota(\Omega)-\iota(d \Omega)$. Remarkably, the correction terms can be algorithmically constructed using only the infinitesimal generators of the group action!

Let $\mathbf{v}_{1}, \ldots, \mathbf{v}_{r} \in \mathfrak{g}$ be a basis for the infinitesimal generators of our transformation group. We adopt the same notation $\mathbf{v}_{\kappa}$ for the prolonged vector field on $\mathrm{J}^{\infty}$ and on $\mathcal{B}^{\infty}=\mathrm{J}^{\infty} \times G$. Let $\mu^{1}, \ldots, \mu^{r}$ be the dual basis for the space of MaurerCartan forms which we view as differential forms on $\mathcal{B}^{\infty}$. Let $\nu^{\kappa}=\sigma^{*} \mu^{\kappa}$ be their pull-backs by the moving frame section. Our key formula is a consequence of the duality between infinitesimal generators and Maurer-Cartan forms, [16]. 
LEMMA 2. If $\Omega$ is any differential form on $\mathrm{J}^{\infty}$, then

$$
d \iota(\Omega)=\iota(d \Omega)+\sum_{\kappa=1}^{r} \nu^{\kappa} \wedge \iota\left[\mathbf{v}_{\kappa}(\Omega)\right]
$$

where $\mathbf{v}_{\kappa}(\Omega)$ denotes the Lie derivative of $\Omega$ with respect to the prolonged infinitesimal generator $\mathbf{v}_{\kappa}$.

We now decompose (3) into invariant horizontal and vertical components. An important observation is that the Lie derivative operation does not — unless the vector field is projectable - preserve the bigrading of our complex. While $\mathbf{v}_{\kappa}$ certainly maps contact forms to contact forms, if $\mathbf{v}_{\kappa}\left(x^{i}\right)=\xi_{\kappa}^{i}(x, u)$, then $\mathbf{v}_{\kappa}\left(d x^{i}\right)=$ $d \xi_{\kappa}^{i}=d_{H} \xi_{\kappa}^{i}+d_{V} \xi_{\kappa}^{i}$ is a combination of horizontal and zero ${ }^{\text {th }}$ order contact forms. Therefore, if $\Omega \in \Omega^{r, s}$, then $\mathbf{v}_{\kappa}(\Omega) \in \Omega^{r, s} \oplus \Omega^{r-1, s+1}$, while $d \Omega \in \Omega^{r+1, s} \oplus \Omega^{r, s+1}$. Consequently, by $(3), d \widetilde{\Omega} \in \widetilde{\Omega}^{r+1, s} \oplus \widetilde{\Omega}^{r, s+1} \oplus \widetilde{\Omega}^{r-1, s+2}$ whenever $\widetilde{\Omega} \in \widetilde{\Omega}^{r, s}$. We decompose the pulled-back Maurer-Cartan forms $\sigma^{*} \mu^{\kappa}=\nu^{\kappa}=\gamma^{\kappa}+\varepsilon^{\kappa}$ into invariant horizontal and invariant contact forms

$$
\gamma^{\kappa}=\sum_{i=1}^{p} C_{i}^{\kappa} \varpi^{i} \in \widetilde{\Omega}^{1,0}, \quad \quad \varepsilon^{\kappa}=\sum_{\alpha, J} E_{\alpha}^{\kappa, J} \vartheta_{J}^{\alpha} \in \widetilde{\Omega}^{0,1} .
$$

The coefficients $C_{i}^{\kappa}, E_{\alpha}^{\kappa, J}$ are certain differential invariants. Substituting into (3) allows us to invariantly decompose the differential $d=d_{\mathcal{H}}+d_{\mathcal{V}}+d_{\mathcal{W}}$, where

$$
\begin{aligned}
& d_{\mathcal{H}} \iota(\Omega)=\iota\left(d_{H} \Omega\right)+\sum_{\kappa=1}^{r} \gamma^{\kappa} \wedge \iota\left(\pi_{r, s}\left[\mathbf{v}_{\kappa}(\Omega)\right]\right), \\
& d_{\mathcal{V}} \iota(\Omega)=\iota\left(d_{V} \Omega\right)+\sum_{\kappa=1}^{r}\left\{\varepsilon^{\kappa} \wedge \iota\left(\pi_{r, s}\left[\mathbf{v}_{\kappa}(\Omega)\right]\right)+\gamma^{\kappa} \wedge \iota\left(\pi_{r-1, s+1}\left[\mathbf{v}_{\kappa}(\Omega)\right]\right)\right\}, \\
& d_{\mathcal{W}} \iota(\Omega)=\sum_{\kappa=1}^{r} \varepsilon^{\kappa} \wedge \iota\left(\pi_{r-1, s+1}\left[\mathbf{v}_{\kappa}(\Omega)\right]\right) .
\end{aligned}
$$

Application of these three fundamental identities will produce all the basic recurrence formulae! In this process, we will repeatedly use the fact that if $F$ is a differential function and $\psi$ is a contact one-form then

$$
d_{\mathcal{H}} F=\sum_{i=1}^{p}\left(\mathcal{D}_{i} F\right) \varpi^{i} \quad d_{\mathcal{H}} \psi=\sum_{i=1}^{p} \varpi^{i} \wedge \mathcal{D}_{i} \psi
$$

Warning: The second identity is not true for a general one-form!

The appearance of the extra differential $d_{\mathcal{W}}$ makes life more complicated, and prevents us from using a lot of the standard bicomplex machinery. Breaking the equation $d^{2}=0$ according to the invariant bigrading leads to the basic formulae

$$
\begin{aligned}
d_{\mathcal{H}}^{2}=0, & d_{\mathcal{H}} d_{\mathcal{V}}+d_{\mathcal{V}} d_{\mathcal{H}}=0,
\end{aligned} \quad d_{\mathcal{V}}^{2}+d_{\mathcal{H}} d_{\mathcal{W}}+d_{\mathcal{W}} d_{\mathcal{H}}=0
$$

We will call such a structure a quasi-tricomplex. If $G$ acts projectably, then $d_{\mathcal{W}}=0$, and $(7)$ reduce to the usual bicomplex relations for $d_{\mathcal{H}}, d_{\mathcal{V}}$, and so the terminology "invariant variational bicomplex" is accurate in this case.

EXAMPLE 3. The Euclidean geometry of plane curves is governed by the standard action $y=x \cos \phi-u \sin \phi+a, v=x \sin \phi+u \cos \phi+b$, of the proper 
Euclidean group $g=(\phi, a, b) \in \mathrm{SE}(2)$ on $M=\mathbf{R}^{2}$. The prolonged group transformations are constructed by applying the implicit differentiation operator $D_{y}=$ $\left(\cos \phi-u_{x} \sin \phi\right)^{-1} D_{x}$ to $v$, and so

$$
v_{y}=\frac{\sin \phi+u_{x} \cos \phi}{\cos \phi-u_{x} \sin \phi}, \quad v_{y y}=\frac{u_{x x}}{\left(\cos \phi-u_{x} \sin \phi\right)^{3}}, \quad \text { etc. }
$$

Solving the normalization equations $y=v=v_{y}=0$ for the group parameters produces the right moving frame

$$
\phi=-\tan ^{-1} u_{x}, \quad a=-\frac{x+u u_{x}}{\sqrt{1+u_{x}^{2}}}, \quad b=\frac{x u_{x}-u}{\sqrt{1+u_{x}^{2}}} .
$$

(The classical moving frame, $[\mathbf{1 3}]$, is the left counterpart obtained by inverting the group element given in (8).) Invariantization of the coordinate functions, which is done by substituting the moving frame formulae into the prolonged group transformations, produces the fundamental normalized differential invariants

$$
\begin{array}{lll}
\iota(x)=H=0, & \iota(u)=I_{0}=0, \quad \iota\left(u_{x}\right)=I_{1}=0, \\
\iota\left(u_{x x}\right)=I_{2}=\kappa, & \iota\left(u_{x x x}\right)=I_{3}=\kappa_{s}, & \iota\left(u_{x x x x}\right)=I_{4}=\kappa_{s s}+3 \kappa^{3},
\end{array}
$$

and so on. The first three, arising from the normalizations, are called phantom invariants. The lowest order non-trivial differential invariant is the Euclidean curvature $I_{2}=\kappa=u_{x x}\left(1+u_{x}^{2}\right)^{3 / 2}$, while $\kappa_{s}, \kappa_{s s}, \ldots$ denote the derivatives of $\kappa$ with respect to the arc-length form $\omega=\sqrt{1+u_{x}^{2}} d x$. The invariant horizontal one-form

$$
\varpi=\iota(d x)=\frac{d x+u_{x} d u}{\sqrt{1+u_{x}^{2}}}=\sqrt{1+u_{x}^{2}} d x+\frac{u_{x}}{\sqrt{1+u_{x}^{2}}} \theta .
$$

is a sum of the contact-invariant arc length form along with a contact correction. In the same manner we obtain the basis invariant contact forms

$$
\vartheta=\iota(\theta)=\frac{\theta}{\sqrt{1+u_{x}^{2}}}, \quad \vartheta_{1}=\iota\left(\theta_{x}\right)=\frac{\left(1+u_{x}^{2}\right) \theta_{x}-u_{x} u_{x x} \theta}{\left(1+u_{x}^{2}\right)^{2}},
$$

The prolonged infinitesimal generators of $\operatorname{SE}(2)$ are

$$
\mathbf{v}_{1}=\partial_{x}, \quad \mathbf{v}_{2}=\partial_{u}, \quad \mathbf{v}_{3}=-u \partial_{x}+x \partial_{u}+\left(1+u_{x}^{2}\right) \partial_{u_{x}}+3 u_{x} u_{x x} \partial_{u_{x x}}+\cdots
$$

The one-forms $\gamma^{\kappa}, \varepsilon^{\kappa}$ governing the correction terms are found by applying the recurrence formulae (5) to the phantom invariants. From the first equation in (5), we obtain

$$
\begin{aligned}
& 0=d_{\mathcal{H}} H=\iota\left(d_{H} x\right)+\iota\left(\mathbf{v}_{1}(x)\right) \gamma^{1}+\iota\left(\mathbf{v}_{2}(x)\right) \gamma^{2}+\iota\left(\mathbf{v}_{3}(x)\right) \gamma^{3}=\varpi+\gamma^{1}, \\
& 0=d_{\mathcal{H}} I_{0}=\iota\left(d_{H} u\right)+\iota\left(\mathbf{v}_{1}(u)\right) \gamma^{1}+\iota\left(\mathbf{v}_{2}(u)\right) \gamma^{2}+\iota\left(\mathbf{v}_{3}(u)\right) \gamma^{3}=\gamma^{2}, \\
& 0=d_{\mathcal{H}} I_{1}=\iota\left(d_{H} u_{x}\right)+\iota\left(\mathbf{v}_{1}\left(u_{x}\right)\right) \gamma^{1}+\iota\left(\mathbf{v}_{2}\left(u_{x}\right)\right) \gamma^{2}+\iota\left(\mathbf{v}_{3}\left(u_{x}\right)\right) \gamma^{3}=\kappa \varpi+\gamma^{3},
\end{aligned}
$$

and hence $\gamma^{1}=-\varpi, \gamma^{2}=0, \gamma^{3}=-\kappa \varpi$. Similarly, applying $d_{\mathcal{V}}$ to the phantom invariants and using the second equation in (5) yields $\varepsilon^{1}=0, \varepsilon^{2}=-\vartheta, \varepsilon^{3}=-\vartheta_{1}$. We are now ready to substitute the non-phantom invariants into (5). The horizontal differentials $d_{\mathcal{H}} I_{k}$ of the normalized differential invariants $I_{n}=\iota\left(u_{n}\right)$ are used to produce the explicit recurrence formulae

$$
\kappa=I_{2}, \quad \kappa_{s}=\mathcal{D} I_{2}=I_{3}, \quad \kappa_{s s}=\mathcal{D} I_{3}=I_{4}-3 I_{2}^{3}, \quad \ldots
$$

relating them to the differentiated invariants $\mathcal{D}^{m} \kappa$. Similarly, the second equation in (5) gives the vertical differential

$$
d_{\mathcal{V}} I_{2}=d_{\mathcal{V}} \kappa=\iota\left(\theta_{2}\right)+\iota\left(\mathbf{v}_{3}\left(u_{x x}\right)\right) \varepsilon^{3}=\vartheta_{2}=\left(\mathcal{D}^{2}+\kappa^{2}\right) \vartheta,
$$


where the final equation follows from the invariant contact form recurrence formulae $\mathcal{D} \vartheta=\vartheta_{1}, \mathcal{D} \vartheta_{1}=\vartheta_{2}-\kappa^{2} \vartheta$, which are found by applying $d_{\mathcal{H}}$ to the invariant contact forms and using the first equation in (5). Finally, applying the second formula in (5) to $\varpi$ yields

$$
d_{\mathcal{V}} \varpi=-\kappa \vartheta \wedge \varpi
$$

\section{Invariant Variational Problems.}

We now apply our construction to derive the formulae for the Euler-Lagrange equations associated with an invariant variational problem. Let us recall the bicomplex construction of the Euler-Lagrange equations. A variational problem $\mathcal{I}[u]=\int L[u] d \mathbf{x}$ is determined by the Lagrangian form $\lambda=L[u] d \mathbf{x} \in \Omega^{p, 0}$. Its differential $d \lambda=d_{V} \lambda \in \Omega^{p, 1}$ defines a form of type $(p, 1)$. We introduce an equivalence relation on such forms, so that $\Theta \sim \Omega$ if and only if $\Theta=\Omega+d_{H} \Psi$ for some $\Psi \in \Omega^{p-1,1}$. The quotient space $\mathcal{F}^{1}=\Omega^{p, 1} / \sim$ is known as the space of source forms. Integration by parts proves that every source form has a canonical representative $\sum_{\alpha=1}^{q} \Delta_{\alpha}\left(x, u^{(n)}\right) \theta^{\alpha} \wedge d \mathbf{x}$, and so can be identified with a $q$-tuple of differential functions $\Delta=\left(\Delta_{1}, \ldots, \Delta_{q}\right)$. In applications, a source form is regarded as defining a system of $q$ differential equations $\Delta_{1}=\cdots=\Delta_{q}=0$ for the $q$ dependent variables $u=\left(u^{1}, \ldots, u^{q}\right)$. Composing the differential $d: \Omega^{p, 0} \rightarrow \Omega^{p, 1}$ with the projection $\pi_{*}: \Omega^{p, 1} \rightarrow \mathcal{F}^{1}$ produces the variational differential $\delta=\pi_{*} \circ$ d that takes a Lagrangian form $\lambda=L[u] d \mathbf{x}$ to its variational derivative source form

$$
\delta \lambda \simeq \sum_{\alpha=1}^{q} \mathbf{E}_{\alpha}(L) \theta^{\alpha} \wedge d \mathbf{x}, \quad \text { where } \quad \mathbf{E}_{\alpha}(L)=\sum_{J}(-D)_{J} \frac{\partial L}{\partial u_{J}^{\alpha}}
$$

are the classical Eulerian (Euler-Lagrange) expressions for the Lagrangian $L$. We extend the definition of the variational derivative $\delta: \Omega^{p} \rightarrow \mathcal{F}^{1}$ to general $p$ forms by setting $\delta \widetilde{\lambda}=\pi_{*} \circ \pi_{p, 1}(\tilde{d})$. Note that $\delta \widetilde{\lambda}=\delta \lambda$ depends only on the horizontal component $\lambda=\pi_{p, 0}(\widetilde{\lambda})$, while annihilating all contact components of $\widetilde{\lambda}$.

According to Lie, $[\mathbf{1 7}, \mathbf{1 8}]$, as long as we work on the regular open subset $\mathcal{V} \subset \mathrm{J}^{\infty}$, any $G$-invariant variational problem is given by an invariant Lagrangian form $\lambda=\widetilde{L} \omega$, where $\omega=\omega^{1} \wedge \cdots \wedge \omega^{p}$ is the contact-invariant volume form, and the invariant Lagrangian $\widetilde{L}$ is an arbitrary differential invariant, and hence a function of the fundamental differential invariants and their invariant derivatives. The associated Euler-Lagrange equations $\mathbf{E}(L)=0$ admit $G$ as a symmetry group, and so, under suitable nondegeneracy hypotheses, [18, Theorem 6.25], can themselves be written in terms of the differential invariants. The problem is to go directly from the differential invariant formula for the variational problem to the differential invariant formula for the Euler-Lagrange equations.

Let us first treat the easier case of curves or one-dimensional submanifolds, so we have only $p=1$ independent variable and $q \geq 1$ dependent variables, where $\operatorname{dim} M=1+q$. In general, the moving frame construction provides us with a certain number, say $\ell$, generating differential invariants $I^{1}, \ldots, I^{\ell}$, such that all higher order differential invariants are obtained by invariant differentiation, $I_{, k}^{\alpha}=\mathcal{D}^{k} I^{\alpha}$, with respect to the contact-invariant one-form $\omega$, which can be viewed as the $G$-invariant arc length element. The comma in the subscript is to remind us that $I_{, k}^{\alpha}$ is not the same as the normalized differential invariant $I_{k}^{\alpha}=\iota\left(u_{k}^{\alpha}\right)$. We use the notation $I^{(n)}$ to denote the collection of all differentiated invariants $I_{, k}^{\alpha}$ up to some prescribed 
order $k \leq n$. It is known, $[\mathbf{1 8}]$, that in most situations $\ell=q$, so there are the same number of generating differential invariants as dependent variables.

A general invariant Lagrangian defines a contact-invariant horizontal one-form $\lambda=\widetilde{L}\left(I^{(n)}\right) \omega \in \Omega^{1,0}$. We replace the contact-invariant Lagrangian form with its fully invariant counterpart $\widetilde{\lambda}=\widetilde{L}\left(I^{(n)}\right) \varpi \in \widetilde{\Omega}^{1,0}$, where $\varpi=\omega+\eta=\iota(d x)$ is the fully invariant one-form obtained by invariantization. We need to compute

$$
d_{\mathcal{V}} \widetilde{\lambda}=d_{\mathcal{V}}(\widetilde{L} \varpi)=d_{\mathcal{V}} \widetilde{L} \wedge \varpi+\widetilde{L} d_{\mathcal{V}} \varpi=\sum_{i, \alpha} \frac{\partial \widetilde{L}}{\partial I_{, i}^{\alpha}} d_{\mathcal{V}} I_{, i}^{\alpha} \wedge \varpi+\widetilde{L} d_{\mathcal{V}} \varpi
$$

We adopt the notation $\Omega \equiv \Theta$ to indicate that the forms $\Omega, \Theta \in \Omega^{p+1}$ have the same source form $\widetilde{\pi_{*}}(\Omega)=\widetilde{\pi_{*}}(\Theta)$, which occurs if and only if $\pi_{p, 1}(\Omega)=\pi_{p, 1}(\Theta)+d_{\mathcal{H}} \Psi$ for some $\Psi \in \widetilde{\Omega}^{p-1,1}$. If $F$ is any differential function and $\psi$ is a contact one-form,

$$
d_{\mathcal{H}}(F \psi)=d_{\mathcal{H}} F \wedge \psi+F d_{\mathcal{H}} \psi, \quad \text { and so } \quad-F d_{\mathcal{H}} \psi \equiv d_{\mathcal{H}} F \wedge \psi .
$$

From (6) it follows that

$$
F(\mathcal{D} \psi) \wedge \varpi \equiv-(\mathcal{D} F) \psi \wedge \varpi .
$$

In particular, if we choose $\psi=d_{\mathcal{V}} H$ for some differential function $H$, then, by (7),

$$
d_{\mathcal{H}} \psi=d_{\mathcal{H}} d_{\mathcal{V}} H=-d_{\mathcal{V}} d_{\mathcal{H}} H=-d_{\mathcal{V}}(\mathcal{D} H \cdot \varpi) .
$$

Therefore, (15) takes the form

$$
F d_{\mathcal{V}}(\mathcal{D} H) \wedge \varpi \equiv-\mathcal{D} F d_{\mathcal{V}} H \wedge \varpi-F(\mathcal{D} H) d_{\mathcal{V}} \varpi
$$

Equations (16), (17) constitute our basic invariant integration by parts formulae. We now iteratively apply (17) to the first term of (14). The first iteration uses $F=\partial \widetilde{L} / \partial I_{, i}^{\alpha}$ and $H=I_{, i-1}^{\alpha}$ so that $\mathcal{D} H=I_{, i}^{\alpha}$. Therefore,

$$
\frac{\partial \widetilde{L}}{\partial I_{, i}^{\alpha}} d_{\mathcal{V}} I_{, i}^{\alpha} \wedge \varpi \equiv-\mathcal{D}\left(\frac{\partial \widetilde{L}}{\partial I_{, i}^{\alpha}}\right) d_{\mathcal{V}} I_{, i-1}^{\alpha} \wedge \varpi-\frac{\partial \widetilde{L}}{\partial I_{, i}^{\alpha}} I_{, i}^{\alpha} d_{\mathcal{V}} \varpi .
$$

Continuing to integrate the first term by parts, we eventually arrive at the formula

$$
d_{\mathcal{V}} \widetilde{\lambda} \equiv \sum_{\alpha=1}^{m} \mathcal{E}_{\alpha}(\widetilde{L}) d_{\mathcal{V}} I^{\alpha} \wedge \varpi-\mathcal{H}(\widetilde{L}) d_{\mathcal{V}} \varpi
$$

where

$$
\mathcal{E}_{\alpha}(\widetilde{L})=\sum_{i=0}^{\infty}(-\mathcal{D})^{i} \frac{\partial \widetilde{L}}{\partial I_{, i}^{\alpha}}, \quad \alpha=1, \ldots, m,
$$

is, by analogy with (13), the invariantized Eulerian of $\widetilde{L}$, while

$$
\mathcal{H}(\widetilde{L})=\sum_{\alpha=1}^{m} \sum_{i>j} I_{, i-j}^{\alpha}(-\mathcal{D})^{j} \frac{\partial \widetilde{L}}{\partial I_{, i}^{\alpha}}-\widetilde{L}
$$

is the invariantized Hamiltonian, which is the counterpart of the usual Hamiltonian

$$
\mathbf{H}(L)=\sum_{\alpha=1}^{m} \sum_{i>j \geq 0} u_{i-j}^{\alpha}\left(-D_{x}\right)^{j} \frac{\partial L}{\partial u_{i}^{\alpha}}-L
$$

associated with a (non-invariant) higher order Lagrangian $L\left(x, u^{(n)}\right)$, cf. $[\mathbf{3}, \mathbf{7}]$. 
In the second phase of the computation, we use the recurrence formulae to compute the vertical differentials

$$
d_{\mathcal{V}} I^{\alpha}=\sum_{\beta=1}^{q} \mathcal{A}_{\beta}^{\alpha}\left(\vartheta^{\beta}\right), \quad d_{\mathcal{V}} \varpi=\sum_{\beta=1}^{q} \mathcal{B}_{\beta}\left(\vartheta^{\beta}\right) \wedge \varpi, \quad \alpha=1, \ldots, m,
$$

in terms of invariant derivatives of the zero ${ }^{\text {th }}$ order invariant contact forms. The $m \times q$ matrix of invariant differential operators $\mathcal{A}=\left(\mathcal{A}_{\beta}^{\alpha}\right)$ will be called the Eulerian operator, while the $1 \times q$ vector of invariant differential operators $\mathcal{B}=\left(\mathcal{B}_{\beta}\right)$ is called the Hamiltonian operator. We finally substitute (22) into (18) and integrate by parts using (16) to obtain the key formula

$$
\begin{aligned}
d \widetilde{\lambda} \equiv \delta \widetilde{\lambda} & =\left(\sum_{\alpha=1}^{m} \sum_{\beta=1}^{q}\left(\mathcal{A}_{\beta}^{\alpha}\right)^{*} \mathcal{E}_{\alpha}(\widetilde{L})-\sum_{\beta=1}^{q}\left(\mathcal{B}_{\beta}\right)^{*} \mathcal{H}(\widetilde{L})\right) \vartheta^{\beta} \wedge \varpi \\
& =\left[\mathcal{A}^{*} \mathcal{E}(\widetilde{L})-\mathcal{B}^{*} \mathcal{H}(\widetilde{L})\right] \vartheta \wedge \varpi,
\end{aligned}
$$

where $\vartheta=\left(\vartheta_{1}, \vartheta_{2}, \ldots, \vartheta_{q}\right)^{T}$. Here ${ }^{*}$ denotes the formal invariant adjoint of an invariant differential operator, so if $\mathcal{P}=\sum_{n} P_{k} \mathcal{D}^{k}$, then $\mathcal{P}^{*}=\sum_{k}(-\mathcal{D})^{k} \cdot P_{k}$. We conclude that the Euler-Lagrange equations for our invariant variational problem are equivalent to the invariant system of differential equations (1).

EXAmple 4. Continuing with Example 3, any Euclidean-invariant variational problem corresponds to a contact invariant Lagrangian $\lambda=\widetilde{L}\left(\kappa, \kappa_{s}, \kappa_{s s}, \ldots\right) \omega$. To compute the Eulerian and Hamiltonian operators we use (22), which, according to (9), (10), take the form $d_{\mathcal{V}} \kappa=\left(\mathcal{D}^{2}+\kappa^{2}\right) \vartheta$ and $d_{\mathcal{V}} \varpi=-\kappa \vartheta \wedge \varpi$. Therefore, the Eulerian operator is $\mathcal{A}=\mathcal{D}^{2}+\kappa^{2}=\mathcal{A}^{*}$, while the Hamiltonian operator $\mathcal{B}=-\kappa=$ $\mathcal{B}^{*}$ is a multiplication operator by $-\kappa$. Both happen to be invariantly self-adjoint. The invariant Euler-Lagrange formula (1) reduces to the known formula, $[\mathbf{3}, \mathbf{1 2}]$,

$$
\left(\mathcal{D}^{2}+\kappa^{2}\right) \mathcal{E}(\widetilde{L})+\kappa \mathcal{H}(\widetilde{L})=0
$$

for the Euclidean-invariant Euler-Lagrange equation.

Let us now tackle the general case of invariant variational problems corresponding to higher dimensional submanifolds. Let $I^{1}, \ldots, I^{\ell}$ denote a fundamental set of differential invariants, which means that the differentiated invariants

$$
I_{, K}^{\alpha}=\mathcal{D}_{\widetilde{K}} I^{\alpha}=\mathcal{D}_{k_{m}} \mathcal{D}_{k_{m-1}} \cdots \mathcal{D}_{k_{1}} I^{\alpha}, \quad \text { where } \quad K=\left(k_{1}, \ldots, k_{m}\right),
$$

contain a complete system of higher order differential invariants. Since the invariant differential operators do not, in general, commute - see $[\mathbf{1 0}, \mathbf{1 6}]$ for the explicit commutation formulae - the order of differentiation is important. The fact that we are allowed to invariantly differentiate $I^{\alpha}$ in any order - not to mention the possible occurrence of additional syzygies among the differentiated invariants, [10], - imply that there can exist many redundancies in our formula for the invariant Lagrangian $\widetilde{\lambda}=\widetilde{L} \varpi$, where $\varpi=\varpi^{1} \wedge \cdots \wedge \varpi^{p}$ is the invariant volume form. Remarkably, these play no significant role in the ensuing computation.

As before we begin by computing

$$
d_{\mathcal{V}} \widetilde{\lambda}=\sum_{\alpha, K} \frac{\partial \widetilde{L}}{\partial I_{, K}^{\alpha}} d_{\mathcal{V}} I_{, K}^{\alpha} \wedge \varpi+\widetilde{L} d_{\mathcal{V}} \varpi
$$


Introduce the $(p-1)$-forms

$$
\left.\varpi_{(i)}=\mathcal{D}_{i}\right\lrcorner \varpi=(-1)^{i-1} \varpi^{1} \wedge \cdots \wedge \varpi^{i-1} \wedge \varpi^{i+1} \wedge \cdots \wedge \varpi^{p} \in \widetilde{\Omega}^{p-1,0} .
$$

If $F$ is any differential function and $\psi$ any contact one-form, then

$$
d_{\mathcal{H}}\left(F \psi \wedge \varpi_{(i)}\right)=d_{\mathcal{H}} F \wedge \psi \wedge \varpi_{(i)}+F d_{\mathcal{H}} \psi \wedge \varpi_{(i)}-F \psi \wedge d_{\mathcal{H}} \varpi_{(i)} .
$$

Since $d_{\mathcal{H}} \varpi_{(i)} \in \widetilde{\Omega}^{p, 0}$, it must be a multiple of the invariant volume form, and we write $d_{\mathcal{H}} \varpi_{(i)}=Z_{i} \varpi$, where $Z_{1}, \ldots, Z_{p}$ are certain differential invariants, which we will call the twist invariants. Using (6) we can rewrite (25) as

$$
F d_{\mathcal{H}} \psi \wedge \varpi_{(i)}=F\left(\mathcal{D}_{i} \psi\right) \wedge \varpi \equiv-\left[\left(\mathcal{D}_{i}+Z_{i}\right) F\right] \psi \wedge \varpi=\left(\mathcal{D}_{i}^{\dagger} F\right) \psi \wedge \varpi,
$$

where $\mathcal{D}_{i}^{\dagger}=-\left(\mathcal{D}_{i}+Z_{i}\right)$ is called the twisted invariant adjoint of the invariant differential operator $\mathcal{D}_{i}$. If we choose $\psi=d_{\mathcal{V}} H$ where $H$ is a differential function, then (26) results in the multivariate invariant integration by parts formula

$$
F d\left(\mathcal{D}_{i} H\right) \wedge \varpi=\left(\mathcal{D}_{i}^{\dagger} F\right) d_{\mathcal{V}} H \wedge \varpi-\sum_{j=1}^{p} F\left(\mathcal{D}_{j} H\right) d_{\mathcal{V}} \varpi^{j} \wedge \varpi_{(i)} .
$$

We use (27) repeatedly to integrate the first term of (24) by parts, leading to

$$
d_{\mathcal{V}} \tilde{\lambda} \equiv \sum_{\alpha=1}^{q} \mathcal{E}_{\alpha}(\widetilde{L}) d_{\mathcal{V}} I^{\alpha} \wedge \varpi-\sum_{i=1}^{p} \mathcal{H}_{j}^{i}(\widetilde{L}) d_{\mathcal{V}} \varpi^{j} \wedge \varpi_{(i)},
$$

where

$$
\mathcal{E}_{\alpha}(\widetilde{L})=\sum_{K} \mathcal{D}_{K}^{\dagger} \frac{\partial \widetilde{L}}{\partial I_{, K}^{\alpha}}, \quad \mathcal{H}_{j}^{i}(\widetilde{L})=-\widetilde{L} \delta_{j}^{i}+\sum_{\alpha=1}^{q} \sum_{J, K} I_{, J, j}^{\alpha} \mathcal{D}_{K}^{\dagger} \frac{\partial \widetilde{L}}{\partial I_{, J, i, K}^{\alpha}},
$$

are, respectively, the invariant Eulerian and invariant Hamiltonian tensor of the invariant Lagrangian $\widetilde{L}$. In (29), we use the twisted adjoints

$$
\mathcal{D}_{K}^{\dagger}=\mathcal{D}_{k_{1}}^{\dagger} \cdots \mathcal{D}_{k_{m}}^{\dagger}=(-1)^{m}\left(\mathcal{D}_{k_{1}}+Z_{k_{1}}\right) \cdots\left(\mathcal{D}_{k_{m}}+Z_{k_{m}}\right), \quad K=\left(k_{1}, \ldots, k_{m}\right),
$$

of the repeated invariant differential operators. Note the reversal in the order of differentiation from that in (23).

The second phase of the computation requires, in analogy with (22), the vertical differentiation formulae

$$
d_{\mathcal{V}} I^{\alpha}=\sum_{\beta=1}^{q} \mathcal{A}_{\beta}^{\alpha}\left(\vartheta^{\beta}\right), \quad \quad d_{\mathcal{V}} \varpi^{j}=\sum_{\beta=1}^{q} \mathcal{B}_{i, \beta}^{j}\left(\vartheta^{\beta}\right) \wedge \varpi^{i},
$$

where $\mathcal{A}=\left(\mathcal{A}_{\beta}^{\alpha}\right)$ denotes the Eulerian operator, which is an $m \times q$ matrix of invariant differential operators, while the $p^{2}$ row vectors $\mathcal{B}_{i}^{j}=\left(\mathcal{B}_{i, \beta}^{j}\right)$ of invariant differential operators form the invariant Hamiltonian operator complex. This allows us to write (28) in the vectorial form

$$
d_{\mathcal{V}} \widetilde{\lambda} \equiv \mathcal{E}(\widetilde{L}) \mathcal{A}(\vartheta) \wedge \varpi-\sum_{i, j=1}^{p} \mathcal{H}_{j}^{i}(\widetilde{L}) \mathcal{B}_{i}^{j}(\vartheta) \wedge \varpi .
$$


We now apply (26) to further integrate both terms by parts. The final result is written in terms of twisted adjoints of Eurlian and Hamiltonian operators,

$$
d_{\mathcal{V}} \widetilde{\lambda} \equiv \delta \widetilde{\lambda}=\left(\mathcal{A}^{\dagger} \mathcal{E}(\widetilde{L})-\sum_{i, j=1}^{p}\left(\mathcal{B}_{i}^{j}\right)^{\dagger} \mathcal{H}_{j}^{i}(\widetilde{L})\right) \vartheta \wedge \varpi .
$$

Proposition 5. The Euler-Lagrange expressions of an invariant Lagrangian form $\widetilde{\lambda}=\widetilde{L}\left(I^{(n)}\right) \varpi$ are equivalent to the invariant system of differential equations

$$
\mathcal{A}^{\dagger} \mathcal{E}(\widetilde{L})-\sum_{i, j=1}^{p}\left(\mathcal{B}_{i}^{j}\right)^{\dagger} \mathcal{H}_{j}^{i}(\widetilde{L})=0
$$

Example 6. Consider the standard action of the Euclidean group $(R, a) \in$ $\mathrm{SE}(3)$ on surfaces $S \subset \mathbf{R}^{3}$. The moving frame computations provide a simple, direct route to the fundamental quantities of Euclidean surface geometry, [13]. It is worth re-emphasizing that all the formulae in this example follow from our infinitesimal moving frame calculus using only linear algebra and differentiation; the explicit formulae for the actual differential invariants (principal curvatures), the Frenet coframe, the invariant contact forms, etc., are never required! We assume that the surface is parametrized by $z=(x, y, u(x, y))$, noting that the final formulae are, in fact, parameter-independent. The classical (local) left moving frame $\rho\left(x, u^{(2)}\right)=(a, R) \in \mathrm{SE}(3)$ consists of the point on the curve defining the translation component $a=z$, while the columns of the rotation matrix $R$ contain the unit tangent vectors forming the Frenet frame along with the unit normal to the surface. The fundamental differential invariants are the principal curvatures $\kappa^{1}=\iota\left(u_{x x}\right), \kappa^{2}=\iota\left(u_{y y}\right)$. The mean and Gaussian curvature invariants $H=\frac{1}{2}\left(\kappa^{1}+\kappa^{2}\right), K=\kappa^{1} \kappa^{2}$, are often used as convenient alternative invariants, since they eliminate some of the residual discrete ambiguities in the moving frame. Higher order differential invariants are obtained by differentiation with respect to the dual invariant differential operators $\mathcal{D}_{1}, \mathcal{D}_{2}$ for the Frenet coframe $\varpi^{1}=\iota\left(d x^{1}\right)$, $\varpi^{2}=\iota\left(d x^{2}\right)$. The differentiated invariants are not functionally independent, since there is a fundamental syzygy

$$
\kappa_{, 22}^{1}-\kappa_{, 11}^{2}+\frac{\kappa_{, 1}^{1} \kappa_{, 1}^{2}+\kappa_{, 2}^{1} \kappa_{, 2}^{2}-2\left(\kappa_{, 1}^{2}\right)^{2}-2\left(\kappa_{, 2}^{1}\right)^{2}}{\kappa^{1}-\kappa^{2}}-\kappa^{1} \kappa^{2}\left(\kappa^{1}-\kappa^{2}\right)=0,
$$

arising from the Codazzi equations. The Codazzi syzygy can, in fact, be directly deduced from our infinitesimal moving frame computations by comparing the recurrence formulae for $\kappa_{, 22}^{1}$ and $\kappa_{, 11}^{2}$ with the normalized invariant $\iota\left(u_{x x y y}\right)$.

Any Euclidean-invariant variational problem has the form $\int \widetilde{L}\left(\kappa^{(n)}\right) \omega^{1} \wedge \omega^{2}$, where $\omega^{1} \wedge \omega^{2}=\pi_{2,0}\left(\varpi^{1} \wedge \varpi^{2}\right)$ is the usual intrinsic surface area 2-form. The invariant Lagrangian $\widetilde{L}$ is an arbitrary differential invariant, and so can be rewritten in terms of the principal curvature invariants and their derivatives, or, equivalently, in terms of the Gaussian and mean curvatures. The former representation leads to simpler formulae and will be retained. From the first formula in (5), we obtain the twist invariants $d_{\mathcal{H}} \varpi_{(1)}=d_{\mathcal{H}} \varpi^{2}=\frac{\kappa_{, 1}^{2}}{\kappa^{1}-\kappa^{2}} \varpi, d_{\mathcal{H}} \varpi_{(2)}=-d_{\mathcal{H}} \varpi^{1}=\frac{\kappa_{, 2}^{1}}{\kappa^{2}-\kappa^{1}} \varpi$, so $Z_{1}=\frac{\kappa_{, 1}^{2}}{\kappa^{1}-\kappa^{2}}, Z_{2}=\frac{\kappa_{, 2}^{1}}{\kappa^{2}-\kappa^{1}}$. The twist invariants appear in Guggenheimer's proof of the fundamental existence theorem for Euclidean surfaces, [13, p. 234]. The 
denominator vanishes at umbilic points on the surface, where the moving frame is not valid. The Codazzi syzygy (32) can be written compactly as

$$
K=\kappa^{1} \kappa^{2}=\mathcal{D}_{1}^{\dagger}\left(Z_{1}\right)+\mathcal{D}_{2}^{\dagger}\left(Z_{2}\right)=-\left(\mathcal{D}_{1}+Z_{1}\right) Z_{1}-\left(\mathcal{D}_{2}+Z_{2}\right) Z_{2},
$$

which expresses the Gaussian curvature $K$ as an invariant divergence. This fact lies at the heart of the Gauss-Bonnet Theorem. The invariant vertical derivatives of the principal curvatures are straightforwardly determined from (5),

$$
\begin{aligned}
& d_{\mathcal{V}} \kappa^{1}=\iota\left(\theta_{x x}\right)=\left(\mathcal{D}_{1}^{2}+Z_{2} \mathcal{D}_{2}+\left(\kappa^{1}\right)^{2}\right) \vartheta \\
& d_{\mathcal{V}} \kappa^{2}=\iota\left(\theta_{y y}\right)=\left(\mathcal{D}_{2}^{2}+Z_{1} \mathcal{D}_{1}+\left(\kappa^{2}\right)^{2}\right) \vartheta,
\end{aligned}
$$

where $\vartheta=\iota(\theta)=\iota\left(d u-u_{x} d x-u_{y} d y\right)$ is the fundamental invariant contact form. Therefore, the Eulerian operator is $\mathcal{A}=\left(\begin{array}{l}\mathcal{D}_{1}^{2}+Z_{2} \mathcal{D}_{2}+\left(\kappa^{1}\right)^{2} \\ \mathcal{D}_{2}^{2}+Z_{1} \mathcal{D}_{1}+\left(\kappa^{2}\right)^{2}\end{array}\right)$. Further,

$$
\begin{aligned}
& d_{\mathcal{V}} \varpi^{1}=-\kappa^{1} \vartheta \wedge \varpi^{1}+\frac{\left(\mathcal{D}_{1} \mathcal{D}_{2}-Z_{2} \mathcal{D}_{1}\right) \vartheta \wedge \varpi^{2}}{\kappa^{1}-\kappa^{2}}, \\
& d_{\mathcal{V}} \varpi^{2}=\frac{\left(\mathcal{D}_{2} \mathcal{D}_{1}-Z_{1} \mathcal{D}_{2}\right) \vartheta \wedge \varpi^{1}}{\kappa^{2}-\kappa^{1}}-\kappa^{2} \vartheta \wedge \varpi^{2},
\end{aligned}
$$

which yields the Hamiltonian operator complex

$\mathcal{B}_{1}^{1}=-\kappa^{1}, \quad \mathcal{B}_{2}^{1}=\frac{1}{\kappa^{1}-\kappa^{2}}\left(\mathcal{D}_{1} \mathcal{D}_{2}-Z_{2} \mathcal{D}_{1}\right)=\frac{1}{\kappa^{1}-\kappa^{2}}\left(\mathcal{D}_{2} \mathcal{D}_{1}-Z_{1} \mathcal{D}_{2}\right)=-\kappa_{1}^{2}$.

Therefore, according to our fundamental formula (5), the Euler-Lagrange equation for a Euclidean-invariant variational problem is

$$
\begin{aligned}
0= & {\left[\left(\mathcal{D}_{1}+Z_{1}\right)^{2}-\left(\mathcal{D}_{2}+Z_{2}\right) \cdot Z_{2}+\left(\kappa^{1}\right)^{2}\right] \mathcal{E}_{1}(\widetilde{L}) } \\
& +\left[\left(\mathcal{D}_{2}+Z_{2}\right)^{2}-\left(\mathcal{D}_{1}+Z_{1}\right) \cdot Z_{1}+\left(\kappa^{2}\right)^{2}\right] \mathcal{E}_{2}(\widetilde{L})+\kappa^{1} \mathcal{H}_{1}^{1}(\widetilde{L})+\kappa^{2} \mathcal{H}_{2}^{2}(\widetilde{L}) \\
& +\left[\left(\mathcal{D}_{2}+Z_{2}\right)\left(\mathcal{D}_{1}+Z_{1}\right)+\left(\mathcal{D}_{1}+Z_{1}\right) \cdot Z_{2}\right] \cdot\left(\frac{\mathcal{H}_{2}^{1}(\widetilde{L})-\mathcal{H}_{1}^{2}(\widetilde{L})}{\kappa^{1}-\kappa^{2}}\right) .
\end{aligned}
$$

As before, $\mathcal{E}_{\alpha}(\widetilde{L})$ are the invariant Eulerians with respect to the principal curvatures $\kappa^{\alpha}$, while $\mathcal{H}_{j}^{i}(\widetilde{L})$ are the invariant Hamiltonians. In particular, if $\widetilde{L}\left(\kappa^{1}, \kappa^{2}\right)$ does not depend on any differentiated invariants, the Euler-Lagrange equation reduces to

$\left[\left(\mathcal{D}_{1}^{\dagger}\right)^{2}+\mathcal{D}_{2}^{\dagger} \cdot Z_{2}+\left(\kappa^{1}\right)^{2}\right] \frac{\partial \widetilde{L}}{\partial \kappa^{1}}+\left[\left(\mathcal{D}_{2}^{\dagger}\right)^{2}+\mathcal{D}_{1}^{\dagger} \cdot Z_{1}+\left(\kappa^{2}\right)^{2}\right] \frac{\partial \widetilde{L}}{\partial \kappa^{2}}-\left(\kappa^{1}+\kappa^{2}\right) \widetilde{L}=0$

For example, the problem of minimizing surface area has invariant Lagrangian $\widetilde{L}=1$, and so has the well-known Euler-Lagrange equation $\mathbf{E}(L)=-\left(\kappa^{1}+\kappa^{2}\right)=$ $-2 H=0$, and hence minimal surfaces have vanishing mean curvature. The mean curvature Lagrangian $\widetilde{L}=H=\frac{1}{2}\left(\kappa^{1}+\kappa^{2}\right)$ has Euler-Lagrange equation

$$
\frac{1}{2}\left[\left(\kappa^{1}\right)^{2}+\left(\kappa^{2}\right)^{2}-\left(\kappa^{1}+\kappa^{2}\right)^{2}\right]=-\kappa^{1} \kappa^{2}=-K=0 .
$$

For the Willmore Lagrangian $\widetilde{L}=\frac{1}{2}\left(\kappa^{1}\right)^{2}+\frac{1}{2}\left(\kappa^{2}\right)^{2},[\mathbf{3}, \mathbf{5}]$, we immediately find the known Euler-Lagrange equation

$$
0=\mathbf{E}(L)=\Delta\left(\kappa^{1}+\kappa^{2}\right)+\frac{1}{2}\left(\kappa^{1}+\kappa^{2}\right)\left(\kappa^{1}-\kappa^{2}\right)^{2}=2 \Delta H+4\left(H^{2}-K\right) H,
$$

where $\Delta=\left(\mathcal{D}_{1}+Z_{1}\right) \mathcal{D}_{1}+\left(\mathcal{D}_{2}+Z_{2}\right) \mathcal{D}_{2}=-\mathcal{D}_{1}^{\dagger} \cdot \mathcal{D}_{1}-\mathcal{D}_{2}^{\dagger} \cdot \mathcal{D}_{2}$ is the Laplace-Beltrami operator on our surface. 
Remark: Anderson, [3], derives the Euler-Lagrange equations for Euclidean surfaces by writing the invariant Lagrangian in terms of the first and second fundamental forms on the surface, whereas, in accordance with our moving frame approach, we write it directly in terms of the intrinsic principal curvature differential invariants. Bryant, [5], uses conformal invariance to construct the Euler-Lagrange equations for the Willmore functional. Implementing our methods for the conformal moving frame will give a formula for the Euler-Lagrange equation of a general conformally-invariant variational problem.

\section{Conclusions.}

In this paper, we have provided a complete, algorithmic solution to the problem of constructing the invariant form of the Euler-Lagrange equations associated with a Lagrangian which admits a finite-dimensional Lie group of variational symmetries. The algorithm relies on the equivariant moving frame method, but only requires the infinitesimal generators, differentiation and linear algebra to construct the required formulae. This is in contrast to the explicit determination of the moving frame and the fundamental differential invariants, which typically requires manipulating rational algebraic expressions, which are notoriously difficult to handle efficiently and accurately in current computer algebra systems.

The general construction of the invariant variational bicomplex based on the equivariant moving frame approach can be applied to a broad range of investigations involving group-invariant quantities appearing in the variational bicomplex, including differential equations, variational problems, conservation laws and characteristic classes. In particular, Anderson and Pohjanpelto, [4], identify the cohomology of the invariant bicomplex with the Lie algebra cohomology of the transformation group. We refer the reader to [16] for more details and additional examples.

Acknowledgments: We would like to thank Ian Anderson, Mark Fels, Vladimir Itskov and Niky Kamran for enlightening discussions on this material.

\section{References}

[1] Anderson, I.M., Introduction to the variational bicomplex, Contemp. Math. 132 (1992), $51-73$.

[2] Anderson, I.M., The Vessiot Handbook, Technical Report, Utah Sate University, 2000.

[3] Anderson, I.M., The Variational Bicomplex, to appear.

[4] Anderson, I.M., and Pohjanpelto, J., The cohomology of invariant of variational bicomplexes, Acta Appl. Math. 41 (1995), 3-19.

[5] Bryant, R.L., A duality theorem for Willmore surfaces, J. Diff. Geom. 20 (1984), 23-53.

[6] Cartan, É., La Méthode du Repère Mobile, la Théorie des Groupes Continus, et les Espaces Généralisés, Exposés de Géométrie No. 5, Hermann, Paris, 1935.

[7] De Donder, T., Théorie Invariantive du Calcul de Variations, Gauthier-Villars, Paris, 1935.

[8] Fels, M., and Olver, P.J., On relative invariants, Math. Ann. 308 (1997), 701-732.

[9] Fels, M., and Olver, P.J., Moving coframes. I. A practical algorithm, Acta Appl. Math. 51 (1998), 161-213.

[10] Fels, M., and Olver, P.J., Moving coframes. II. Regularization and theoretical foundations, Acta Appl. Math. 55 (1999), 127-208.

[11] Griffiths, P.A., On Cartan's method of Lie groups and moving frames as applied to uniqueness and existence questions in differential geometry, Duke Math. J. 41 (1974), 775-814.

[12] Griffiths, P.A., Exterior Differential Systems and the Calculus of Variations, Progress in Math. vol. 25, Birkhäuser, Boston, 1983. 
[13] Guggenheimer, H.W., Differential Geometry, McGraw-Hill, New York, 1963.

[14] Itskov, V., Orbit reduction of exterior differential systems, and group-invariant variational problems, preprint, University of Minnesota, 2000.

[15] Jensen, G.R., Higher order contact of submanifolds of homogeneous spaces, Lecture Notes in Math., No. 610, Springer-Verlag, New York, 1977.

[16] Kogan, I.A., and Olver, P.J., Invariant Euler-Lagrange equations and the invariant variational bicomplex, preprint, University of Minnesota, 2000.

[17] Lie, S., Über Integralinvarianten und ihre Verwertung für die Theorie der Differentialgleichungen, Leipz. Berichte 49 (1897), 369-410; also Gesammelte Abhandlungen, vol. 6, B.G. Teubner, Leipzig, 1927, pp. 664-701.

[18] Olver, P.J., Equivalence, Invariants, and Symmetry, Cambridge University Press, Cambridge, 1995.

[19] Olver, P.J., Moving frames and singularities of prolonged group actions, Selecta Math. 6 (2000), 41-77.

[20] Rund, H., The Hamilton-Jacobi Theory in the Calculus of Variations, D. Van Nostrand Co. Ltd., Princeton, N.J., 1966 .

[21] Tsujishita, T., On variational bicomplexes associated to differential equations, Osaka J. Math. 19 (1982), 311-363.

[22] Tulczyjew, W.M., The Lagrange complex, Bull. Soc. Math. France 105 (1977), 419-431.

[23] Vinogradov, A.M., The $\mathcal{C}$-spectral sequence, Lagrangian formalism and conservation laws. I. The linear theory, J. Math. Anal. Appl. 100 (1984), 1-40.

[24] Vinogradov, A.M., The $\mathcal{C}$-spectral sequence, Lagrangian formalism and conservation laws. II. The nonlinear theory, J. Math. Anal. Appl. 100 (1984), 41-129.

Department of Mathematics, Yale University, New Haven, Connecticut 06520

E-mail address: kogan@math.yale.edu

School of Mathematics, University of Minnesota, Minneapolis, MN 55455

E-mail address: olver@ima.umn.edu 